\title{
Influence of Road Traffic Noise on English Reading Comprehension of Chinese College Students Majoring in English
}

\author{
https://doi.org/10.3991/ijet.v15i14.15355 \\ Wei Ding \\ Heilongjiang Bayi Agricultural University, Daqing, China \\ dingwei0508@gmail.com
}

\begin{abstract}
Noise has a negative impact on language learning. But first and second languages are affected differently by noise. To clarify the effects of road traffic noise on second language learning, this study explores deep into how road traffic noise, the main noise on university campuses, affects the English reading comprehension of Chinese college students majoring in English. The subjects were asked to finish English reading comprehension tests of three difficulty levels (easy, medium and hard) under road traffic noises of three sound pressure levels (SPLs), namely, $30 \mathrm{dBA}, 50 \mathrm{dBA}$ and $70 \mathrm{dBA}$. The score, time consumption and number of interruptions of each subject were recorded, and subjected to one-way analysis of variance (one-way ANOVA). In addition, the subjects were asked to evaluate the impacts of road traffic noises on their comprehension. The results show that: the scores of English reading comprehension gradually decreased with the growing of the SPL of road traffic noise, but the decrease is only significant for easy questions; the time consumption of reading comprehension was not greatly affected by the SPL of road traffic noise; the subjects were interrupted more frequently by road traffic noises of $50 \mathrm{dBA}$ and $70 \mathrm{dBA}$ than road traffic noise of $30 \mathrm{dBA}$, but the difference is only significant for hard test; according to the self-evaluation of the English majors, interruption is the main problem brought by road traffic noise, which is replaced by distraction at the SPL of $70 \mathrm{dBA}$. Overall, the road traffic noise can disturb the English reading comprehension of Chinese college students majoring in English, and the disturbance is related to the difficulty of the text. The higher the SPL of the road traffic noise, the readers are more anxious and more frequently disturbed in reading, and the lower the score of English comprehension. In addition, attention should be paid to the disturbance of $30 \mathrm{dBA}$ road traffic noise on second language learners.
\end{abstract}

Keywords-Highway traffic noise, sound pressure level (SPL), English reading comprehension, difficulty, Chinese college students

\section{Introduction}

Along with the development of urbanization, the noise has an increasingly serious impact on life. Noise exceeding a certain limit will have a variety of negative effects 
on human physiology and psychology. The evidence shows that environmental noise has adverse effects on cognition, mental health, quality of life, and even leads to many diseases [1]. Animal experiments have also indicate that long-term high noise exposure will cause a decline in learning and memory ability, which may be related to the impairment of the long-term potentiation effect of brain neurons [2-3]. Howard et al. [4] found that children under the common signal-to-noise ratio in the classroom need to make more efforts to concentrate if they want to hear the teaching content.

The survey shows that Chinese campuses are generally subject to noise pollution, and outdoor noise is dominated by road traffic noise pollution [5-11]. Highway traffic noise can distract students' attention, induce emotional changes, affect learning effects, and damage mental health. Even if below the national standard, the traffic noise is still considered by the interviewed students as the most undesirable outdoor noise category [6]. The impact of traffic noise on student learning has gradually become the focus of research.

So far, the research results of noise interference in learning haven't been consistent. Through the literature analysis of Clark and Paunovic [12], it's found that the impacts of traffic noise on short-term memory and attention vary in different studies. Various factors such as the noise source, SPL, the type of learning, and the age of the subject all influence the consistency of the study results.

The adverse effect of highway noise on college students' reading increases with the increase of SPL [5, 13]. Wang et al. conducted a psychological test of the effects of noise on eighth graders and found that when the noise level exceeded $55 \mathrm{~dB}$, the adverse effects were significantly increased, concluding that $55 \mathrm{~dB}$ was the largest classroom acceptable noise level in psychology [14]. Studies have also shown that noise of $50 \mathrm{~dB}$ or more can interfere with college students' learning [13, 15]. However, Qi and Hou [13] believe that $30-65 \mathrm{~dB}$ of noise only has a psychological impact on college students. Pan et al. found through research that different noise levels have no difference in college students' digital short-term memory, and simple learning tasks are not affected by the type of noise and its SPL, but with the SPL increasing, the subjects still need more effort to concentrate on the learning [16].

Studies on test subjects of different ages have shown that there are age differences in recognition of noise disturbing syllable; children's cognitive performance of consonants is worse than vowels and is more affected by noise [17]. The influence of noise on short-term memory is more significant in young children, and the influence on auditory memory is greater than that of visual memory [18]. However, some other studies have indicated that the impact of noise on plot and semantic memory (the ability to perform tasks) has nothing to do with age [19].

The influence of noise on learning is also related to the type and difficulty of learning tasks. Studies on children have shown that the degree of annoyance caused by noise increases with the increase in cognitive complexity [20]. Boman et al. [19] conducted research on subjects of different ages and found that the episodic memory with high difficulty level is more affected by traffic noise than semantic memory. During discussion and teaching, students' recognition of sentences is not affected by noise, but their comprehension ability decreases significantly with the increase of noise [21]. Higher noise levels will lead to weaker use of vocabulary and phonology during read- 
ing and writing [22]. The impacts of noise from different levels of highways on college students' foreign language reading are significantly different, but not on native language reading [5]. Studies for the impact of noise on auditory short-term memory also show that the performance of second language vocabulary is worse than that of first language [23]. Besides, it's found through the research on classroom transformation that improving the sound insulation conditions can significantly promote the students' achievements, among which English and other language subjects have a much higher performance than the mathematics and other subjects that can be learned through vision [24].

Previous studies on the influence of noise on college students have shown conflicting results. Apart from acoustic factors, they may be mainly caused by different test types. Language learning, especially foreign languages, may be more disturbed by noise. Noises above $50 \mathrm{dBA}$ adversely affect college students' reading speed and English reading comprehension [13]. Jiang's research on Chinese college students has shown that highway noise is more likely to distract subjects' attention during English reading than reading in their native language; the impact of road noise on reading is mainly reflected in the subjective feelings of the subjects, and may have no effect on test score [5]. Other studies also indicate that although traffic noise affects subjective annoyance, it has no effect on test scores [20]. Peng and Wang [25] studied American college students and found that the performance of non-native students with poor English ability in understanding English is more subjected to the background noise level. Thus, the difficulty degree significantly affects the interference of noise in foreign language learning. Clark and Sörqvist [26] believe that there are two different types of auditory interference: one is the process interruption caused by attracting attention, and the other is the interference in the process. The difficulty of foreign language materials may affect the type of noise interference, and especially process interference may bring greater difficulties to text understanding.

In view of the above, this paper attempts to better explore the influence of traffic noise on foreign language learning. For this, taking Chinese college students majoring in English as research subjects, reading texts of different difficulty were selected, to evaluate the impact of different SPL traffic noise on the reading process and reading scores. The survey was conducted on the subjective feelings of the respondents in order to understand the cause of noise interference.

\section{Methods}

\subsection{Participants}

A total of 18 students participated in this study. They came from the third year of English major at Heilongjiang Bayi Agricultural University, and all passed the College English Test (CET 6). All participants had normal hearing, normal vision or corrected vision. 


\subsection{Materials and apparatus}

The traffic noise of the city's main road, with a small amount of whistle sounds, was used in the test. The audio file was in MP3 format, downloaded from www.aigei.com, and the duration was 77 seconds. The SPL of the noise was set to three levels of $30 \mathrm{dBA}, 50 \mathrm{dBA}$, and $70 \mathrm{dBA}$. During the test, each subject was in a separate room, using 2 speakers (in front, placed in a regular triangle with the subject, at a distance of $1 \mathrm{~m}$ ) for loop playback. SPL is the average value of the entire audio frequency with an error of $\pm 1 \mathrm{dBA}$.

There were three sets of English test questions for reading comprehension test. Each set contains reading comprehension questions at three levels of difficulty-PART ONE (Easy), PART TWO (Medium), and PART THREE (Hard); each part includes 10 choice questions. The difficulty and completion time made no difference between different sets of test questions ( $p>0.1$ ). Table 1 lists the differences between test questions of different difficulty levels.

Table 1. Scores and time consumption of test questions of different difficulty levels

\begin{tabular}{|l|c|c|c|c|}
\hline \multicolumn{1}{|c|}{ Difficulty Level } & Easy & Medium & Hard & p-value \\
\hline Scores & $7.53 \pm 0.21$ & $6.51 \pm 0.21$ & $6.06 \pm 0.23$ & 0.00 \\
\hline Time $(\mathrm{s})$ & $868.06 \pm 38.86$ & $937.55 \pm 28.65$ & $1089.3 \pm 30.7$ & 0.00 \\
\hline
\end{tabular}

\subsection{Design and procedure}

The test was carried out from February 25 to 27, 2020. The test time was 09:0010:30. The doors and windows were closed when answering questions and artificial light sources were used to ensure even light in the area where the subject is located.

The subjects completed different test questions under the road traffic noise environment of a specific SPL for three consecutive days. In order to avoid the errors caused by the test time, the SPL and test questions of the daily subjects were randomly allocated through the computer to ensure that there are two subjects with different combinations of SPL and test question every day. A total of 54 test samples were finally obtained-18 for each SPL noise environment.

The test recorded the score, time consumption, and number of interruptions (reading is interrupted and need to be repeated) of each Part completed by each subject, and subjectively scored the noise annoyance (0-3; 0 means no annoyance; 1 means the mood is slightly affected and not calm; 2 means emotional anxiety; 3 means extreme annoyance and inability to concentrate). After each test, the subjects answered the questionnaire, including whether the reading was disturbed, and the reason for the interference-"distraction" (attention is interfered through the processes) or "interruption" (attention being captured by the sound, and reread is needed).

\subsection{Data analysis}

Z-score standardized processing was performed on each subject's score, time consumption, number of interruptions, and annoyance. Considering that the absolute 
value of the standardized data is less than 2 , the abnormal data corresponding to some testers were eliminated, so that the overall data conforms to the normal distribution.

One-way ANOVA (IBM SPSS statistics 22.0) was used to analyze the influence of highway traffic noise SPL on the scores, time consumption, number of interruptions, and annoyance of English majors in English reading. Meanwhile, the difficulty of reading materials was divided into three groups (Easy, Medium, Hard). Thus, when considering the English reading comprehension of different difficulty, the influences of the SPL analyzed using the One-Way ANOVA differ in terms of the score, time consumption, interruptions, and annoyance.

The statistics were conducted about the frequency of each option in the questionnaire such as reading interference and the related reasons for the subject under different SPL noises. Chi Square Test (IBM SPSS statistics 22.0) was performed to analyze the impact of road traffic noise on the self-evaluation of English majors in English reading comprehension, and make sure whether there are differences under different SPLs.

The statistical result data is expressed in Means \pm SD. In the figures, * represents a significant difference $(\mathrm{p}<0.05)$, and $* *$ represents a very significant difference $(\mathrm{p}<0.01)$.

\section{$3 \quad$ Result and Discussion}

\subsection{Scores of the reading comprehension}

Fig. 1 shows that as the SPL of road traffic noise increased, the English reading comprehension scores of Chinese college students gradually decreased, and the reading score under $70 \mathrm{dBA}$ noise was significantly $(\mathrm{p}<0.05)$ lower than at $30 \mathrm{dBA}$. Considering the difficulty of reading materials, the reading comprehension scores of different difficulties all decreased with the increase of SPL, but the impact of traffic noise of different SPL on the English reading scores of Chinese college students is only significant for easy questions (Fig. 2), that is, $70 \mathrm{dBA}$ and $30 \mathrm{dBA}$ levels of SPL noises have a significant effect on reading achievement $(\mathrm{p}<0.05)$.

Therefore, the adverse effect of noise on foreign language reading comprehension is related to the text difficulty. Only simple reading test questions can achieve better results in a quieter environment. However, this test did not set up a quiet environment with no traffic noise. There are no significant differences in reading results between the $30 \mathrm{dBA}$ and $50 \mathrm{dBA}$ noise groups for reading tests of different difficulty levels, which may be because the $30 \mathrm{dBA}$ traffic noise is already in a quiet environment, but still interfere with the subjects compared to SPL life noise. 


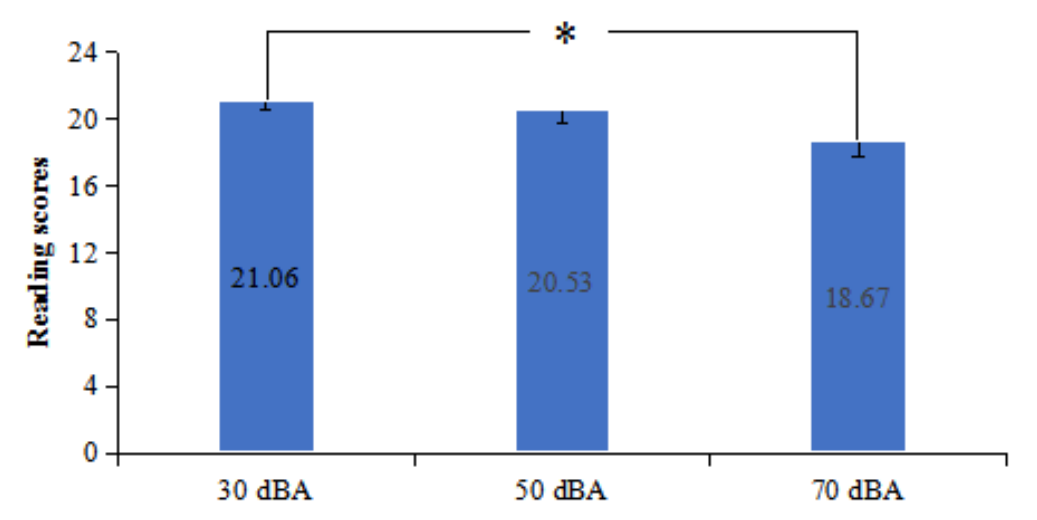

Fig. 1. English reading scores of Chinese undergraduates at different traffic noise levels

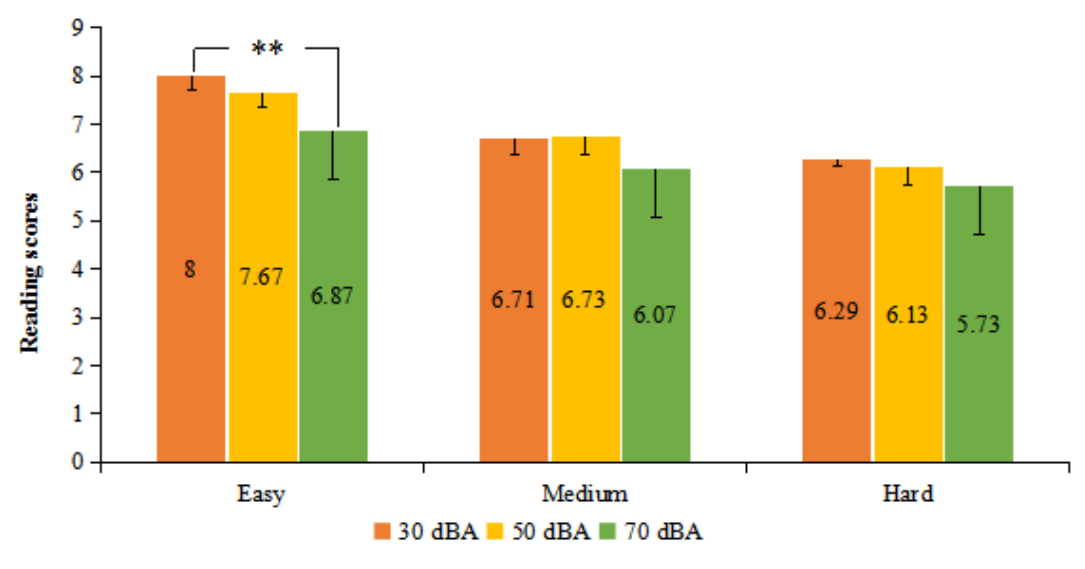

Fig. 2. Effect of SPLs on scores of English reading at different difficulty levels

Notes: Different superscript letters $(a-b)$ indicate that variables within a row are significantly different $(\mathrm{P}<0.05)$.

\subsection{Time consumption for the reading comprehension}

As shown in Fig. 3, the SPL of road traffic noise had no significant effect on Chinese college students' English reading comprehension time $(p>0.10)$. The results were similar when distinguishing the difficulty of reading materials. The time for the subjects to complete the reading comprehension of three difficulty levels was not affected by the noise SPL (Fig. 4). This may be due to the good English ability of the subjects so that they can overcome the interference and complete the reading under the three noise environments tested. 


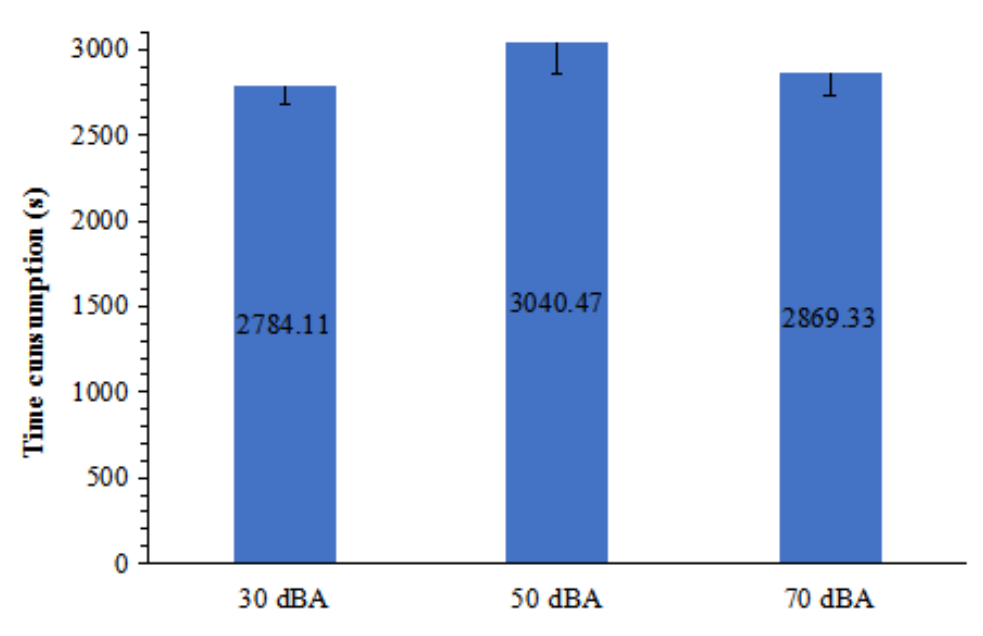

Fig. 3. Time consumption for English reading of Chinese undergraduates at different traffic noise levels

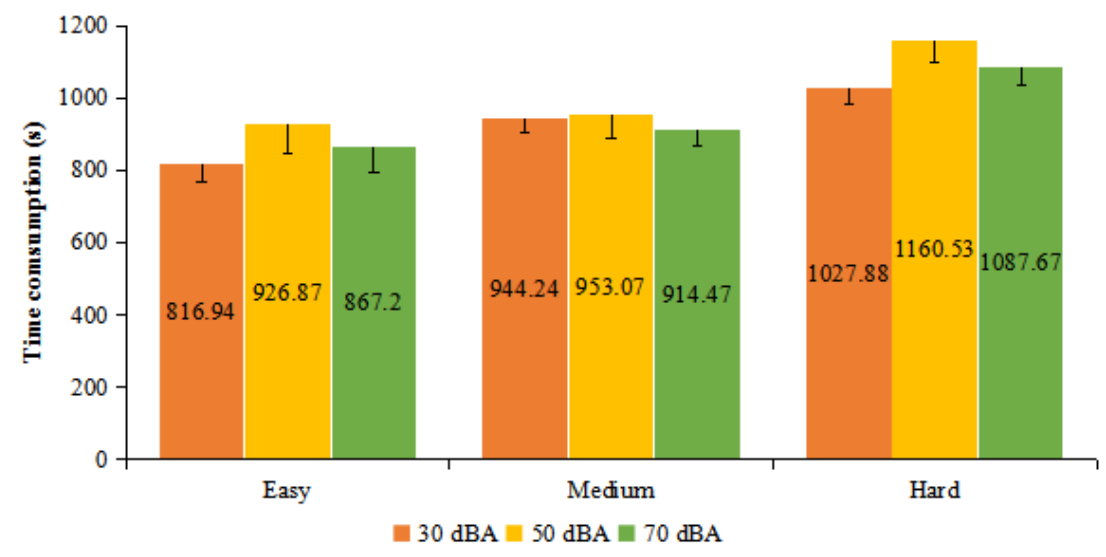

Fig. 4. Effect of SPLs on time consumption of English reading at different difficulty levels

\subsection{Number of interruptions}

Fig. 5 shows that compared with $30 \mathrm{dBA}, 50 \mathrm{dBA}$ and $70 \mathrm{dBA}$ traffic noise interrupted college English reading more frequently, but the difference was not significant $(\mathrm{p}=0.18)$. Fig. 6 shows the influence of noise SPL when using the reading materials at different difficulty level: for hard questions, $70 \mathrm{dBA}$ of traffic noise causes a reading interruption more significantly than $30 \mathrm{dBA}(\mathrm{p}<0.05)$. Reading interruption may be caused by harsh sounds such as whistle in traffic noise, and its SPL is significantly higher, so the interruption caused by $30 \mathrm{dBA}$ level noise may be less. However, for 
the easy/medium text, the subjects are more likely to overcome the interference, but prone to interrupt the reading by the harsh noise when reading the hard text.

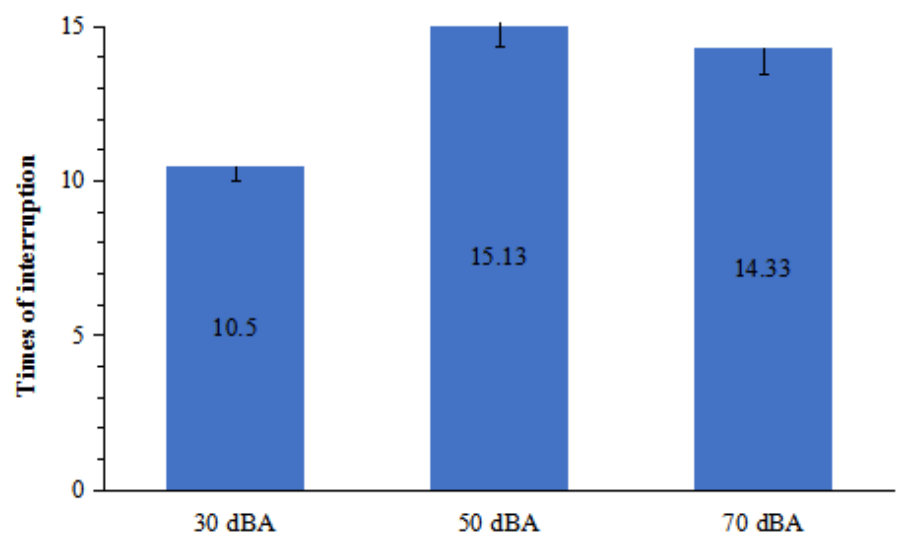

Fig. 5. Times of interruptions during English reading of Chinese undergraduates at different traffic noise levels

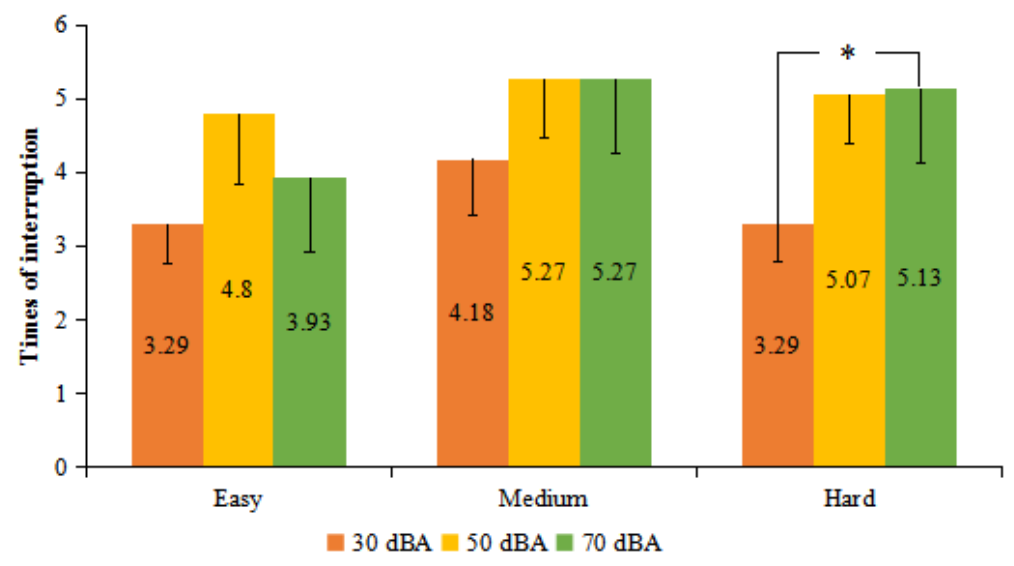

Fig. 6. The effect of SPLs on interruptions of English reading at different difficulty levels

Notes: Different superscript letters $(\mathrm{a}-\mathrm{b})$ indicate that variables within a row are significantly different $(\mathrm{P}<0.05)$.

\subsection{Self-evaluated annoyance to noise}

Fig. 7 shows that with the SPL of road traffic noise increasing, the annoyance of Chinese college students in the process of English reading comprehension gradually increased. Compared with $30 \mathrm{dBA}$ noise, the increase in student annoyance under 70 
dBA noise was extremely significant (uncalm vs. restless, $\mathrm{p}<0.01$ ). For easy/medium texts, significant differences only exist between the $30 \mathrm{dBA}$ noise group and the 70 dBA noise group (Easy: $\mathrm{p}<0.05$; Medium: $\mathrm{p}<0.01$ ), as shown in Fig. 8. For hard text, the level of annoyance caused by traffic noise was significantly different among the three SPL groups ( $\mathrm{p}<0.01$, see Fig. 8).

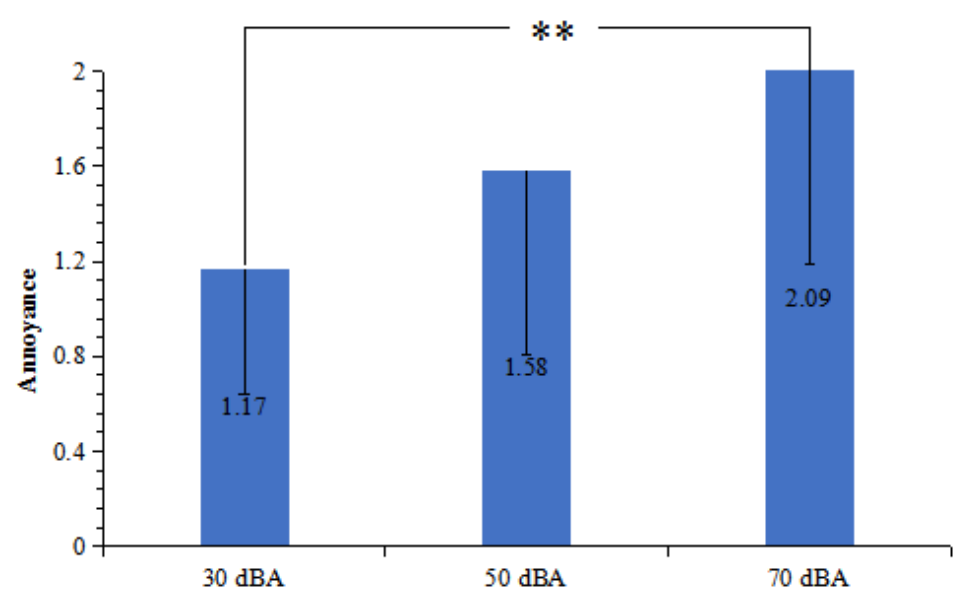

Fig. 7. Annoyance of Chinese undergraduates in English reading at different traffic noise levels

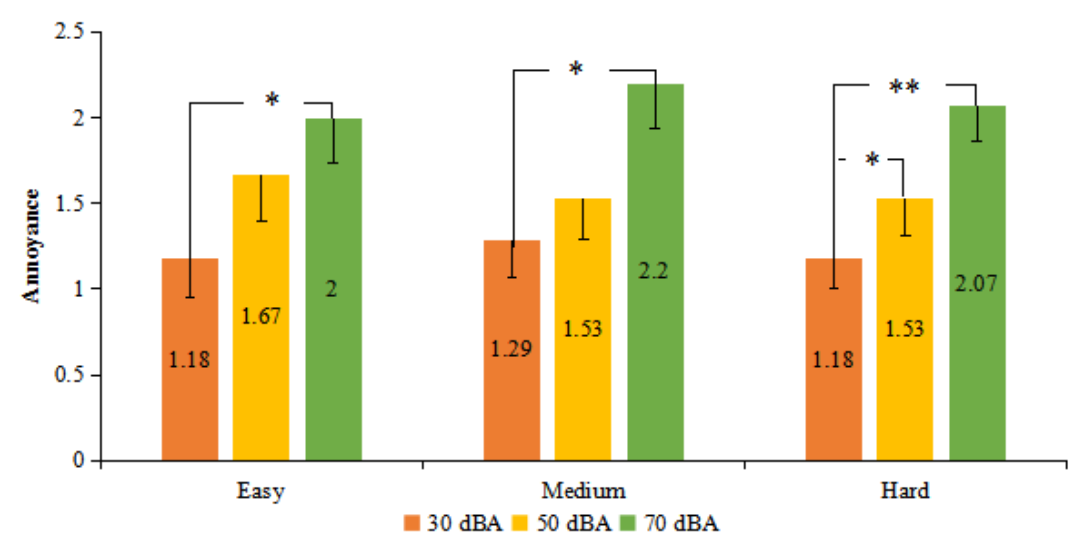

Fig. 8. Effect of SPLs on the annoyance to noises in English reading at different difficulty levels

Notes: Different superscript letters $(\mathrm{a}-\mathrm{b})$ indicate that variables within a row are significantly different $(\mathrm{P}<0.05),(\mathrm{A}-\mathrm{B})$ indicate that variables within a row are very significantly different $(\mathrm{P}<0.01)$. 
Therefore, $70 \mathrm{dBA}$ traffic noise annoys college students more seriously, which will interfere with the thinking and important information memory during reading. This does not result in increased reading time, but the students may be more likely to be confused by the wrong choice in answering the question, which is consistent with the difference in reading scores caused by SPL. When reading hard text, the annoyance changed more significantly with SPL, indicating that that the psychology may be easily interfered by environmental noise in face of more difficult tasks.

As shown in Fig. 9, under $50 \mathrm{dBA}$ and $70 \mathrm{dBA}$ noises, more college students believed that they were disturbed by road traffic noise during the reading test $(p<0.05)$. This is consistent with the results of noise SPL on reading scores and annoyance. However, under $30 \mathrm{dBA}$ noise, $63 \%$ of the subjects considered to be disturbed, which means that quiet traffic noise may also cause general disturbance to non-native English readers.

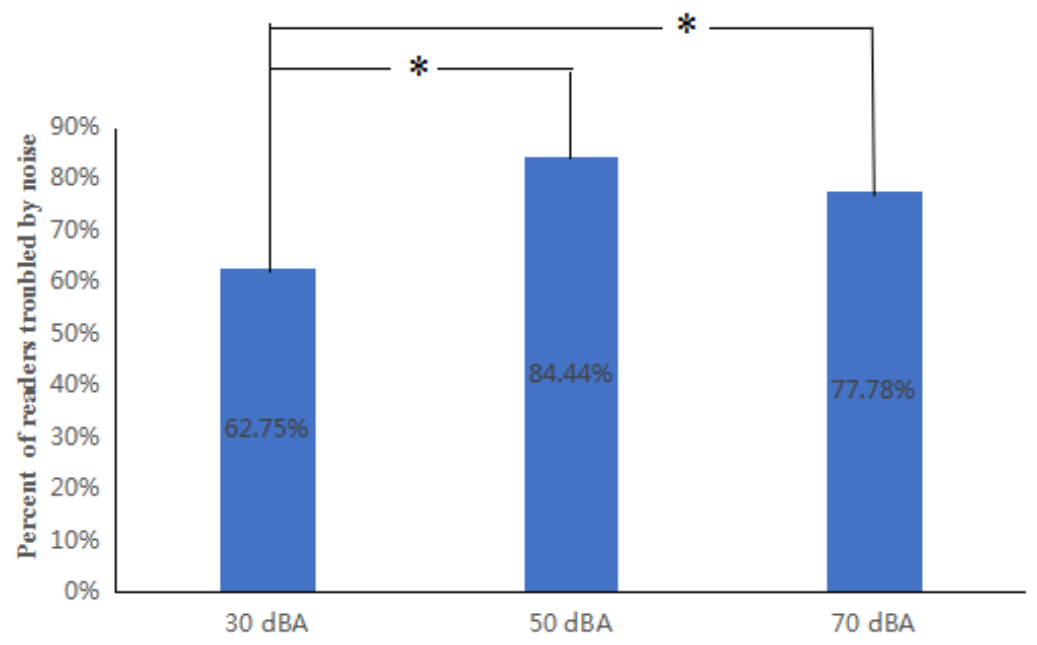

Fig. 9. Effect of SPLs on percent of readers troubled by noises

In the self-evaluation, more college students thought that the noise interference was "interruption" rather than "distraction" (59.05\% vs. 40.95\%). However, under $70 \mathrm{dBA}$ noise, "distraction" is the main cause of interference (Fig. 10), which is in contradiction to the fact that reading is interrupted more frequently at $70 \mathrm{dBA}$ noise, but consistent with the subject's increased anxiety. Therefore, although the high SPL traffic noise causes attention to be attracted and interrupts reading, it continues to interfere with emotions and fails to concentrate. It has a greater impact on college students' second language reading comprehension. 


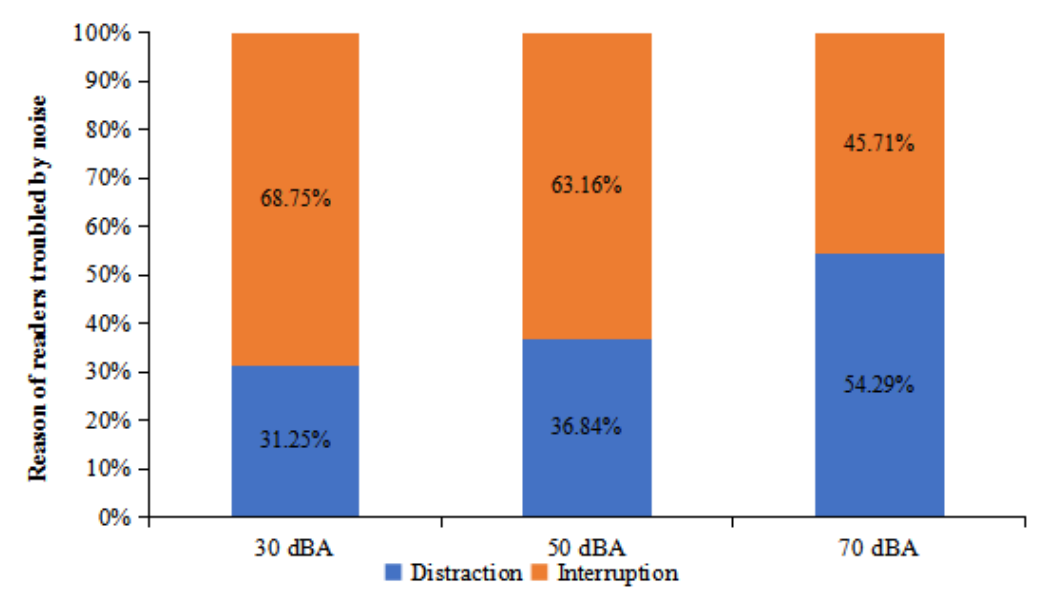

Fig. 10. Effect of SPLs on the reason of readers troubled by noises

\section{Conclusion}

For Chinese college students majoring in English reading comprehension, the degree of annoyance gradually increases with the SPL of road traffic noise, and more students believe that reading is disturbed. This does not result in increased reading time, but reducing the reading performance.

The adverse effect of noise on foreign language reading comprehension is related to the difficulty of the text. Only easy reading can achieve significantly better results in a quieter environment. For hard text, reading is interrupted more frequently under high noise. Psychology may be easily disturbed by environmental noise when completing more difficult reading, thus causing interruption of reading.

$63 \%$ of the subjects thought that $30 \mathrm{dBA}$ of road traffic noise also disturbed their reading, indicating that the adverse effect of "quiet" level of traffic noise should also receive more attention.

Interrupted reading under low-to-medium-level noise conditions is the main feature of noise interference. Under the noise of $70 \mathrm{dBA}$, despite the increase in reading interruption caused by noise and the subjects' annoyance, "distraction" has also become the main factor affecting college students' second language reading comprehension.

\section{$5 \quad$ References}

[1] Clark, C., Crumpler, C., Notley, H. (2020). Evidence for Environmental Noise Effects on Health for the United Kingdom Policy Context: A Systematic Review of the Effects of Environmental Noise on Mental Health, Wellbeing, Quality of Life, Cancer, Dementia, Birth, Reproductive Outcomes, and Cognition. International Journal of Environmental Research and Public Health, 17(2): 393. https://doi.org/10.3390/ijerph17020393 
[2] Tang, Y., Yuan, H., Zhang, X., Tu, B. (2008). The influence of noise on learning memory behaviors in juvenile rats and expression of GLU positive neurons in hippocampus. Modern Preventive Medicine, 35(04): 668-670

[3] Tang, Y., Cheng, S., Yuan, H., Tu, B. (2008). The influence of noise on learning memory behaviors in and expression of GABA neurons in hippocampus. Journal of Chongqing Medical University, 33(5): 557-559.

[4] Howard, C.S., Munro, K.J., Plack, C.J. (2010). Listening effort at signal-to-noise ratios that are typical of the school classroom. International Journal of Audiology, 49(12), 928932. https://doi.org/10.3109/14992027.2010.520036

[5] Jiang, Y. (2018). Effects of road noises on college students' English and Chinese reading. Huaqiao University.

[6] Song, Y., Sheng, S., Zhu, P. (2007). Exterior and Interior Noise Survey of Classrooms of Tongji University. Journal of Tongji University (Nature Science), 35(3): 372-376.

[7] Niu, Z., Niu, F., Chen, L., Feng, Q. (2010). Measurement and accessment of campus environmental noise in Yan'an University. Journal of Yanan University (Nature Science Edition), 29(3): 58-61. https://doi.org/10.3969/j.issn.1004-602X.2010.03.018

[8] Yu, H., Asiya, K., Liu, L. (2011). Measurement and assessments of campus environmental noise in Xinjiang normal university. Journal of Xinjiang Normal University (Natural Sciences Edition), 30(3): 21-24. https://doi.org/10.3969/j.issn.1008-9659.2011.03.005

[9] Gao, A., Li, A., Fang, X., Zhu, Z., Li, L. (2015). The evaluation on the campus environmental noise in Shijiazhuang university of economics and the study on control measures. Shanxi Science and Technology, 1: 91-92

[10] Di, X. (2015). Environment noise monitoring and evaluation of college of information, Shanxi agricultural university campus. Journal of Shanxi Normal University (Natural Science Edition), 29(04): 124-128

[11] Liang, L., Zhou, G., Jiang, X., Xiao, P.P., Xiao, L. (2014). Analysis of noise of Chongshan campus of Liaoning university. Journal of Liaoning University (Nature Science Edition, 41(2): 176-182. https://doi.org/10.3969/j.issn.1000-5846.2014.02.016

[12] Clark, C. Paunovic, K. (2018). WHO environmental noise guidelines for the European region: A systematic review on environmental noise and cognition. International Journal of Environmental Research and Public Health, 15(2): 285. https://doi.org/10.3390/ijerph1502 $\underline{0285}$

[13] Qi, P., Hou, C. (2000). Effect of environmental noise on students' self-study efficiency, Women-Environment.Health-the Symposium on Women and Environment, Beijing, China. 147-149.

[14] Wang, J., Shi, Q., Zhong, X. (1992). Effect of traffic noise on classroom behavior of primary and middle school students. Acta Acustica, 4: 248-255

[15] Yang, J., Liu, B., Ling, L., Li, Z., Shao, X., Tang, Y. (2014) Effect of Noise on College Students' Attention. Journal of Environmental \& Occupational Medicine, 31(2): 119-121, 125.

[16] Pan, X., Meng, Z. (2009). Effect of noise interference on the attention of digital short-term memory, 2009 National Conference on Environmental Acoustics, Beihai, China. 59-61.

[17] Johnson, C.E. (2000). Children's Phoneme Identification in Reverberation and Noise. Journal of Speech, Language, and Hearing Research, 43(1): 144-157. https://doi.org/10.10 44/jslhr.4301.144

[18] Peng, J. Jiang, P. (2016). Effects of noise and reverberation on children's visual short-term memory. Journal of South China University of Technology (Natural Science Edition), 44(07): 130-134. 
[19] Boman, E., Enmarker, I., Hygge, S. (2005). Strength of noise effects on memory as a function of noise source and age. Noise \& Health, 7(27): 11-26. https://doi.org/10.4103/1463-1 $\underline{741.31636}$

[20] Zhang, L. Ma, H. (2018). Effects of environment noise on children's short-term memory and attention stability. Acta Acustica, 43(2): 246-252.

[21] Valente, D., Plevinsky, H., Franco, J.M., Heinrichsgraham, E.C., Lewis, D.E. (2012). Experimental investigation of the effects of the acoustical conditions in a simulated classroom on speech recognition and learning in children. Journal of the Acoustical Society of America, 2131(1): 232-246. https://doi.org/10.1121/1.3662059

[22] Santos, J.F., Souza, A.P., Seligman, L. (2013). Comparative analysis of performance in reading and writing of children exposed and not exposed to high sound pressure levels. CoDAS, 25(3): 274-281. https://doi.org/10.1590/S2317-17822013000300014

[23] Hurtig, A., De Poll, M.K., Pekkola, E., Hygge, S., Liung, R., Sörqvist, P. (2016). Children's recall of words spoken in their first and second language: effects of signal-to-noise ratio and reverberation time. Frontiers in Psychology, 6: 2029. https://doi.org/10.3389/fpsy g.2015.02029

[24] Li, J., Fan, G., Kenneth P.R. (2010). Study on the effect of classroom acoustic environment on student learning, 2010 National Membership Congress and Academic Conference of Chinese Acoustics Society, Harbin, China. 298-299

[25] Peng, Z., Wang, L. (2016). Effects of noise, reverberation and foreign accent on native and non-native listeners' performance of English speech comprehension. Journal of the Acoustical Society of America, 139(5): 2772-2783. https://doi.org/10.1121/1.4948564

[26] Clark, C., Sörqvist, P. (2012). A 3-year update on the influence of noise on performance and behavior. Noise Health, 14(61): 292-296. https://doi.org/10.4103/1463-1741.104896

\section{$6 \quad$ Author}

Wei Ding is a lecturer in Department of English, College of Humanities and Social Sciences, Heilongjiang Bayi Agricultural University. She is interested in English education and British literature. Email: dingwei0508@gmail.com

Article submitted 2020-05-01. Resubmitted 2020-06-17. Final acceptance 2020-06-20. Final version published as submitted by the authors. 